\title{
Evaluación de una Técnica de PCR-Múltiple para la Detección Rápida de Salmonella Typhimurium y Enteritidis en Cuyes (Cavia porcellus) Naturalmente Infectados
}

\author{
Evaluation of a Multiplex-PCR Method for Rapid Detection of Salmonella \\ Typhimurium and Enteritidis in Naturally Infected Guinea Pigs (Cavia porcellus)
}

Gerardo Díaz O. ${ }^{1}$, Raúl Rosadio A. ${ }^{1}$, Geraldine Marcelo M. ${ }^{1}$, Ana Chero O. ${ }^{1}$, Ronald Jiménez A. ${ }^{2}$, Iván Reyna W. ${ }^{3}$, Lenin Maturrano H. ${ }^{1,45}$

\section{Resumen}

El objetivo del estudio fue evaluar la capacidad de detección de Salmonella Typhimurium y Enteritidis mediante el método de la Reacción en Cadena de la Polimerasa (PCR) Múltiple a partir de muestras en pre-enriquecimiento no selectivo (utilizando secuencias blanco de los genes InvA, fliC y prot6E), y hallar su concordancia con el método de detección microbiológica convencional, que consta de pre-enriquecimiento no selectivo, enriquecimiento selectivo, aislamiento en agar diferencial y pruebas bioquímicas. Se analizaron 111 muestras de hígado de cuyes con diagnóstico presuntivo de salmonelosis, provenientes de Chancay (Lima) y El Mantaro (Junín), Perú. Se llegó a detectar Salmonella Typhimurium por PCR Múltiple en 54\% (60/111) de las muestras y en $41 \%$ (45/111) por análisis microbiológico. Se encontró una concordancia substancial con un valor de Kappa de 0.64. La prueba de McNemar demostró que los resultados de ambas pruebas son estadísticamente diferentes $(\mathrm{p}<0.05)$.

Palabras clave: PCR múltiple; detección rápida; análisis microbiológico; Salmonella, cobayo

\section{AbSTRACT}

The aim of this study was to evaluate the detection capacity of the Multiplex Polymerase Chain Reaction (PCR) method from non-selective pre-enrichment samples (using target sequences of InvA, fliC and prot6E genes) to diagnose Salmonella

\footnotetext{
${ }^{1}$ Laboratorio de Microbiología y Parasitología Veterinaria, ${ }^{2}$ Instituto de Investigaciones Tropicales y de Altura (IVITA El Mantaro), ${ }^{3}$ Instituto de Investigaciones Tropicales y de Altura (IVITA Huaral), ${ }^{4}$ Laboratorio de Zootecnia y Producción Agropecuaria, Facultad de Medicina Veterinaria, Universidad Nacional Mayor de San Marcos, Lima, Perú

${ }^{5}$ E-mail: lenin.maturrano@gmail.com
} 
Typhimurium and Enteritidis and to determine the concordance between the conventional microbiological detection method which consist of non-selective pre-enrichment, selective enrichment, differential agar isolation and biochemical tests. A total of 111 liver samples from guinea pigs with presumptive diagnosis of salmonellosis, collected in Chancay (Lima) and El Mantaro (Junín), Peru were analyzed. Salmonella Typhimurium was detected by multiplex PCR in 54\% (60/111) of the samples and by microbiological analysis in $41 \%$ (45/111). A substantial concordance was found with a Kappa value of 0.64. The McNemar test showed that the results of both tests were statistically different $(p<0.05)$.

Key words: multiplex PCR; rapid detection; microbiological isolation; Salmonella; guinea pig

\section{INTRODUCCIÓN}

El cuy (Cavia porcellus) es una especie nativa de los andes sudamericanos que cuenta con una población aproximada de 13.6 millones (INEI, 2012). Su importancia como animal de producción y sus ventajas económicas son indiscutibles, ya que es una fuente alimenticia de alto valor para las poblaciones del Perú, contribuyendo a la seguridad alimentaria del país (Chauca, 1997).

Diversos problemas sanitarios afectan seriamente la crianza del cuy. Entre ellos, los brotes de salmonelosis pueden afectar entre el 61 y 95\% de las granjas (Ramírez, 1972; Matsuura et al., 2010), siendo los serovares Typhimurium y Enteritidis los más aislados (Fox et al., 1984). La importancia de esta enfermedad radica en su rápida evolución, que conlleva a una alta mortalidad y disminución de la productividad (Chauca, 2007).

El método convencional de detección de Salmonella spp es el análisis microbiológico a través de un enriquecimiento no selectivo, enriquecimiento selectivo, aislamiento en agar diferencial y confirmación mediante pruebas bioquímicas y serotipificación (ISO, 2002; FDA, 2007), procedimientos que pueden tomar hasta una semana. Por otro lado, se dispone de un protocolo de PCR-Múltiple para identificar Salmonella Typhimurium y
Salmonella Enteritidis aislados de cuyes (Jamshidi et al., 2010; Marcelo, 2015), el cual amplifica simultáneamente fragmentos de 284 $\mathrm{pb}$ del gen $\operatorname{Inv} A$ que codifica una invasina específica del género Salmonella (Rahn et al., 1992), fragmentos de $559 \mathrm{pb}$ del gen fliC que codifica para la flagelina $\mathrm{H} 1$ específica del serovar Typhimurium (Soumet et al., 1999) y fragmentos de $185 \mathrm{pb}$ del gen Prot6E que codifica para una fimbria específica del serovar Enteritidis (Clavijo et al., 2006). Asimismo, otros estudios (Matias et al., 2010; Zhang et al., 2011; Mainar-Jaime et al., 2013) han demostrado que se puede identificar Salmonella mediante PCR después del pre-enriquecimiento no selectivo de las muestras.

Es de gran importancia contar con un método de detección de Salmonella Typhimurium y Enteritidis que sea práctico y consuma menos tiempo para poder tomar medidas de control inmediatas, a fin de contrarrestar las considerables pérdidas económicas que trae la presentación de esta enfermedad en la producción de cuyes. Por ello, el presente estudio tuvo como objetivo evaluar la capacidad de detección de Salmonella Typhimurium y Enteritidis de la PCR Múltiple a partir de muestras sometidas a pre-enriquecimiento no selectivo y hallar la concordancia entre esta y el método convencional de análisis microbiológico de detección de Salmonella spp. 


\section{Materiales y Métodos}

\section{Lugar y Tiempo}

La toma de muestras se llevó a cabo en la estación experimental «El Mantaro» del Instituto Veterinario de Investigaciones de Trópico y Altura (IVITA) de la Facultad de Medicina Veterinaria, Universidad Nacional Mayor de San Marcos (FMV-UNMSM) (Jauja, Perú), y en un criadero de crianza comercial en Chancay (Huaral, Perú). El procesamiento de las muestras se llevó a cabo en el laboratorio de Genética y Biología Molecular de la FMV-UNMSM en Lima. Ambas etapas se ejecutaron entre octubre de 2015 y abril de 2016.

\section{Toma de Muestras}

Se analizaron 111 cuyes (13 del IVITA y 98 del criadero de Chancay), de 1 día a 20 meses de edad, muertos con signos de depresión, pelaje hirsuto, anorexia, caquexia, diarrea y postración; signos desencadenantes de muerte súbita y compatibles con diagnóstico presuntivo de salmonelosis (Chauca, 1997). Los especímenes completos fueron colectados y llevados al laboratorio, siguiendo los lineamientos establecidos por el Servicio Nacional de Sanidad Agraria del Perú (SENASA, 2014), para realizar la necropsia dentro de las $24 \mathrm{~h}$ post mortem y tomar como muestras de referencia el parénquima hepático (Matsuura, 2008; Layme, 2009).

\section{Procesamiento de las Muestras}

Las muestras fueron analizadas en simultáneo siguiendo dos vías: análisis microbiológico y PCR-múltiple a partir del pre-enriquecimiento no selectivo (PENS), como se observa en la Figura 1. Para el análisis microbiológico se adaptó el protocolo de la norma ISO-6579:2002 (ISO, 2002) y el manual para análisis bacteriológico de Salmonella (FDA, 2007), siguiendo las etapas de preenriquecimiento no selectivo en Agua Peptonada Tamponada (APT) por $18 \mathrm{~h}$ a $37^{\circ} \mathrm{C}$ (de donde se obtuvo $1 \mathrm{ml}$ para el análisis por PCR múltiple), enriquecimiento selectivo en medio Rappaport-Vassiliadis (RV) por $24 \mathrm{~h}$ a $42{ }^{\circ} \mathrm{C}$, aislamiento diferencial en agar selectivo Xilosa Lisina Desoxicolato (XLD) por $24 \mathrm{~h}$ a $37^{\circ} \mathrm{C}$ y caracterización bioquímica por Agar Hierro Triple Azúcar (TSI), Agar Lisina Hierro (LIA), Agar Sulfito Indol Motilidad (SIM), Agar Citrato de Simmons y Agar Urea por $24 \mathrm{~h}$ a $37^{\circ} \mathrm{C}$.

Para el PCR-múltiple se trabajó con 1 $\mathrm{ml}$ de APT obtenido a partir de la etapa de PENS, se extrajo el ADN total con el kit comercial GeneJET Genomic DNA Purification Kit (Thermo Fisher Scientific) y se realizó la PCR múltiple siguiendo el protocolo de Jamshidi et al. (2010), con los cebadores indicados en el Cuadro 1. Se utilizó $1 \mathrm{X}$ Buffer, $2 \mathrm{mM} \mathrm{MgCl}, 1 \mathrm{mM}$ dNTP's, mezcla de $1 \mu \mathrm{M}$ de cebadores, $1 \mathrm{U}$ Taq polimerasa, $3 \mu 1$ de $\mathrm{ADN}$ y $\mathrm{H}_{2} \mathrm{O}$ hasta un volumen final de $20 \mu$, siguiendo las condiciones estandarizadas por Marcelo (2015) de 35 ciclos de desnaturalización por $45 \mathrm{~s}$ a $95^{\circ} \mathrm{C}$, hibridación por $45 \mathrm{~s}$ a $58{ }^{\circ} \mathrm{C} \mathrm{y}$ elongación por $45 \mathrm{~s}$ a $72{ }^{\circ} \mathrm{C}$, con una desnaturalización inicial de $5 \min$ a $95^{\circ} \mathrm{C} \mathrm{y}$ una elongación final de $7 \mathrm{~min}$ a $72^{\circ} \mathrm{C}$. Los productos de la PCR-múltiple fueron separados mediante electroforesis en gel de agarosa al $2 \%$, con TBE $0.5 \mathrm{X}$ como buffer de corrida, a $100 \mathrm{~V}$ por $2 \mathrm{~h}$. Se tiñó el gel con bromuro de etidio y se visualizaron las bandas mediante transiluminador de luz ultravioleta. Como controles positivos de la PCR se utilizaron cepas referencia de Salmonella Typhimurium (ATCC 14028) y Salmonella Enteritidis (ATCC 13076).

\section{Análisis de Datos}

Los resultados fueron analizados a partir de una tabla de contingencia $2 \times 2$. Se utilizó la prueba de Kappa para determinar el grado de concordancia (Cuadro 2) entre los dos métodos y la prueba de McNemar para determinar si los resultados eran estadísticamente similares a un nivel de significancia del 5\% (González y Falcón, 1999). 


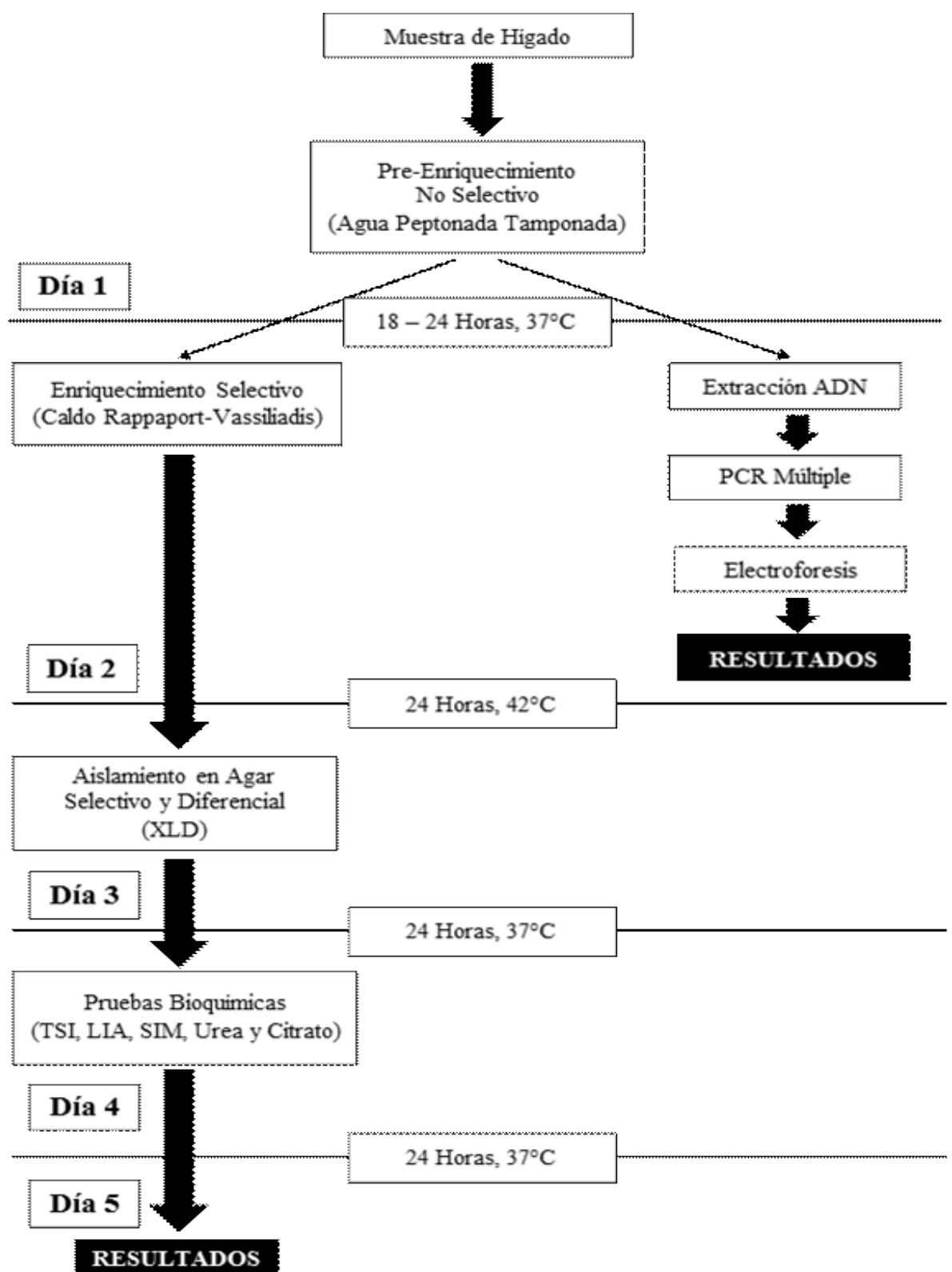

Figura 1. Diagrama de flujo del procesamiento de muestras para la detección de Salmonella spp mediante análisis microbiológico y análisis molecular

\section{Resultados}

Se analizaron 111 muestras de parénquima hepático de cuyes. Mediante el análisis microbiológico se detectaron 45 animales positivos a Salmonella spp (41\%) y mediante la PCR-múltiple se detectaron 60 positivos a Salmonella Typhimurium (54\%) (Cuadro 3). El producto de la PCR en el gel electroforesis se observa en la Figura 2.

El índice de Kappa fue de $\mathrm{K}=0.64$, lo que indica un grado de concordancia substancial. Por otro lado, mediante la prueba de McNemar se obtuvo un $\mathrm{X}_{\mathrm{Mc}}=9.8$, lo cual 
Cuadro 1. Cebadores empleados en la PCR-Múltiple para identificar a Salmonella Typhimurium y Enteritidis (Jamshidi et al., 2010)

\begin{tabular}{|c|c|c|c|c|}
\hline Bacteria & Gen & Secuencia del cebador 5'-3' & $\begin{array}{c}\text { Producto } \\
(\mathrm{pb})\end{array}$ & Referencia \\
\hline $\begin{array}{l}\text { Salmonella } \\
\text { spp }\end{array}$ & $\operatorname{inv} A$ & $\begin{array}{c}\text { GTGAAATTATCGCCACGTTCGGGCAA } \\
\text { TCATCGCACCGTCAAAGGAACC }\end{array}$ & 284 & $\begin{array}{l}\text { Rahn et al. } \\
\quad(1992)\end{array}$ \\
\hline $\begin{array}{l}\text { Salmonella } \\
\text { Typhimurium }\end{array}$ & $f l i C$ & $\begin{array}{l}\text { CGGTGTTGCCCAGGTTGGTAAT } \\
\text { ACTCTTGCTGGCGGTGCGACTT }\end{array}$ & 559 & $\begin{array}{l}\text { Soumet et } \\
\text { al. (1999) }\end{array}$ \\
\hline $\begin{array}{l}\text { Salmonella } \\
\text { Enteritidis }\end{array}$ & Prot6E & $\begin{array}{l}\text { ATATCGTCGTTGCTGCTTCC } \\
\text { CATTGTTCCACCGTCACTTTG }\end{array}$ & 185 & $\begin{array}{l}\text { Clavijo et al. } \\
\quad(2006)\end{array}$ \\
\hline
\end{tabular}

Cuadro 2. Grados de concordancia para Kappa (Landis y Koch, 1977)

\begin{tabular}{cc}
\hline Valor de K & Grado de concordancia \\
\hline$<0$ & Muy pobre \\
$0-0.20$ & Ligera \\
$0.21-0.40$ & Regular \\
$0.41-0.60$ & Moderada \\
$0.61-0.80$ & Substancial \\
$0.81-1.00$ & Perfecta \\
\hline
\end{tabular}

indica que los resultados de ambos métodos fueron estadísticamente diferentes y no se pueden reemplazar mutuamente.

\section{Discusión}

La detección de Salmonella spp puede realizarse por diversos métodos, especialmente por PCR y el análisis microbiológico (OIE, 2010; García, 2011), siendo este último el «gold standard» en los laboratorios de referencia (ISO, 2002; FDA, 2007; OIE, 2010). Tiene como principal desventaja su baja sensibilidad y el tiempo que demanda para obtener resultados (González et al., 2014). Por otro lado, la PCR es una herramienta analítica de mayor sensibilidad que las propias pruebas «gold standard», por lo que su uso está en camino de sustituir los métodos de detección clásicos (OIE, 2008). De lo expuesto se deduce que es crucial contar con un método de detección de Salmonella que sea práctico y consuma menos tiempo para poder tomar medidas de control más rápidas.

Los resultados obtenidos muestran que es posible detectar con éxito Salmonella Typhimurium mediante PCR-múltiple a partir de muestras de hígado de cuyes en preenriquecimiento, lográndose detectarla en $54 \%(60 / 111)$ de los animales, mientras que mediante la microbiología convencional solo se logró detectar Salmonella spp en el $41 \%$ (45/111) de los animales; es decir, el PCRmúltiple detectó un $25 \%$ más de muestras positivas que la microbiología convencional. Por otro lado, la concordancia hallada entre ambos métodos fue substancial $(\mathrm{K}=0.64)$; sin embargo, los resultados de las pruebas fueron estadísticamente diferentes entre sí, lo que significa que no pueden utilizarse indistintamente para detectar Salmonella spp debido a que una tiene mayor sensibilidad que la otra.

Uno de los motivos del mayor porcentaje de detección de Salmonella spp mediante la PCR-múltiple fue que se realizó des- 


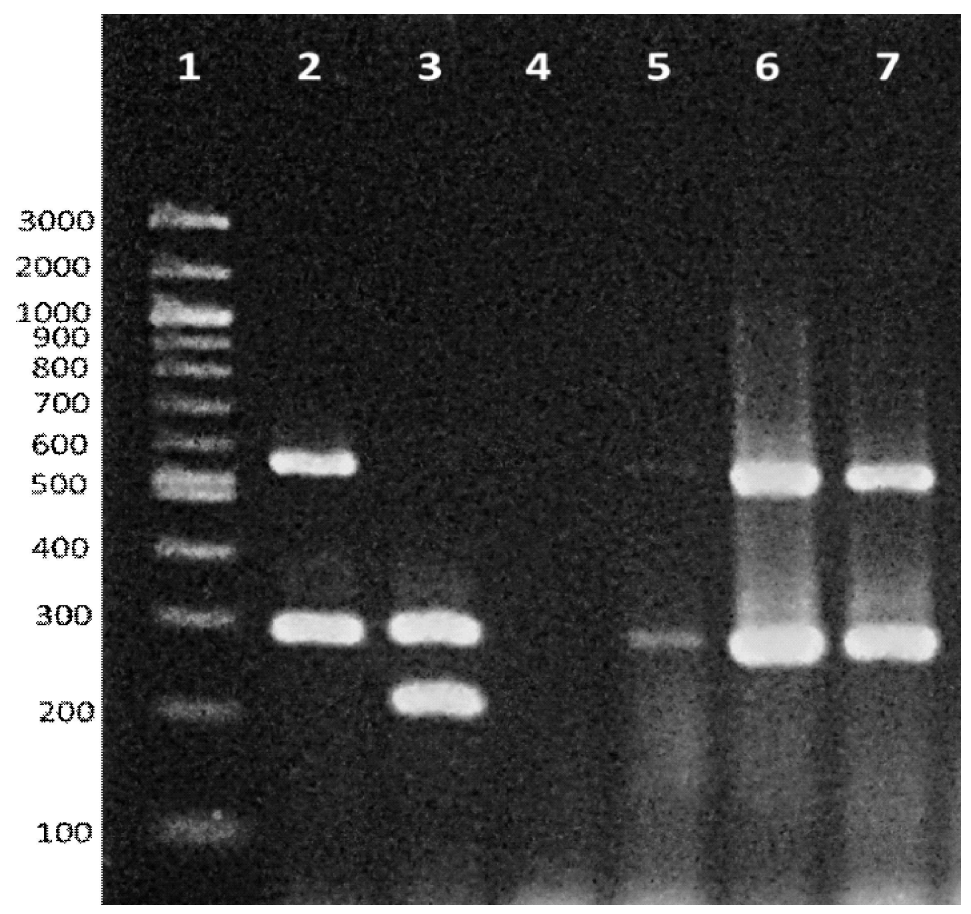

Figura 2. Electroforesis en gel de agarosa $2 \%$ de los productos de la PCR-Múltiple para Salmonella Typhimurium y Enteritidis. Carril 1: Marcador molecular $100 \mathrm{pb}$. Carril 2: Control positivo (Salmonella Typhimurium, ATCC 14028). Carril 3: Control positivo (Salmonella Enteritidis, ATCC 13076). Carril 4: Control negativo (agua ultrapura). Carril 5 a 7: Muestras positivas a Salmonella Typhimurium

Cuadro 3. Resultados de detección de Salmonella mediante análisis microbiológico y PCRmúltiple distribuidos en una tabla de contingencia de $2 \times 2$

\begin{tabular}{llccc}
\hline & & \multicolumn{3}{c}{ Análisis microbiológico } \\
\cline { 3 - 5 } & & Positivo & Negativo & Total \\
\hline Análisis & Positivo & 44 & 16 & 60 \\
molecular & Negativo & 1 & 50 & 51 \\
\hline Total & & 45 & 66 & 111 \\
\hline
\end{tabular}

pués del pre-enriquecimiento no selectivo, el cual es necesario para reactivar la Salmonella que contenga la muestra; factor que fue de gran importancia en otros estudios (Eriksson y Aspan, 2007; Kumar et al., 2007; Matias et al., 2010; Zhang et al., 2011; Mainar-Jaime et al., 2013); mas no crucial, ya que otros autores se refieren a la etapa de enriquecimiento selectivo mediante MSRV (Medio Rappaport-Vassiliadis Semi-Solido), principalmente porque diluye las sustancias inhibidoras de la PCR y reduce la carga bacteriana competitiva en la muestra, aunque significa un día más para obtener el re- 
sultado (Ferraz-Castagna et al., 2005; Myint et al., 2006; Eriksson y Aspan., 2007; Soria et al., 2012). Por otro lado, las evidencias para la detección mediante PCR directa de muestras sin pre-enriquecimiento son contradictorias y no muy alentadoras, principalmente debido a las bajas cargas y a la presencia de inhibidores (Ocepek et al., 2006; Matias et al., 2010; Marathe et al., 2012).

En el desempeño y concordancia de la PCR en muestras de pre-enriquecimiento respecto al análisis microbiológico convencional se obtienen sensibilidades que varían desde 85 al $98 \%$ y una concordancia substancial con índice de kappa de aproximadamente 0.76 (Feder et al., 2001; FerrazCastagna et al., 2005; Myint et al., 2006; Kumar et al., 2007), lo que coincide con el rango hallado en este estudio. Por otro lado, el límite de diagnóstico en muestras artificialmente contaminadas es muy variable, siendo como mínimo $2 \mathrm{UFC} / \mathrm{ml}$ y de $10^{3} \mathrm{UFC} / \mathrm{ml}$ como máximo, según al protocolo de PCR (Ocepek et al., 2006; Zhang et al., 2011; Kanki et al., 2012; González et al., 2014), llegando en algunos casos a poderse detectar Salmonella spp únicamente mediante PCR (Ahmadi et al., 2010).

Una ventaja importante del protocolo de PCR utilizado en este estudio es que amplifica la secuencia blanco del gen InvA, el cual ha sido ampliamente utilizado en este tipo de estudios (Ferraz-Castagna et al., 2005; Eriksson y Aspan, 2007; Kumar et al., 2007; Ahmadi et al., 2010; Zhang et al., 2011; Soria et al., 2012) y que está validado como estándar para la detección molecular de Salmonella spp (Malorny et al., 2003). Cabe destacar este aspecto, ya que se tuvo una muestra positiva al diagnóstico microbiológico, pero no al molecular, lo cual pudo deberse a errores en el pipeteo o por efecto de algún inhibidor de la PCR. Para evitar estos falsos negativos y tener un mejor control de calidad del protocolo de PCR, se recomienda el uso de controles de amplificación interno (Ferraz-Castagna et al., 2005; OIE, 2008; Zhang et al., 2011).
Las razones de una menor detección de Salmionella spp por el análisis microbiológico convencional se deberían principalmente a la viabilidad de las bacterias, y a la recolección, el transporte y la cantidad de muestra (García, 2011), ya que este método necesita bacterias viables, al contrario de la PCR que solo requiere la presencia de $\mathrm{ADN}$ (Pérez et al., 2008).

\section{Conclusión}

- El presente protocolo de PCR-múltiple permite detectar a Salmonella Typhimurium en dos días, a partir de muestras en pre-enriquecimiento no selectivo, tomadas de cuyes muertos con diagnóstico presuntivo de salmonelosis.

- La concordancia del PCR-múltiple respecto al análisis microbiológico convencional es substancial $(\mathrm{K}=0.64)$, pero los resultados de ambos métodos fueron estadísticamente diferentes y no se pueden reemplazar mutuamente.

\section{Agradecimientos}

Este estudio fue financiado por el Programa Nacional de Innovación para la Competitividad y Productividad - Innóvate Perú, del Proyecto Contrato 362-PNICPPIAP-2014 «Desarrollo de una vacuna para el control y prevención de la salmonelosis en la producción cuyes».

\section{Literatura Citada}

1. Ahmadi M, Dalirnaghadeh B, Aski H, Khoshbakht R. 2010 Comparison of polymerase chain reaction (PCR) and conventional cultivation methods for detection of carriers of Salmonella spp. in cattle and buffalo. Comp Clin Pathol 19: 251-255. doi: 10.1007/s00580-009$0867-\mathrm{y}$ 
2. Chauca L. 1997. Producción de cuyes (Cavia porcellus). Rome: Organización de las Naciones Unidas para la Agricultura y la Alimentación. [Internet]. Disponible en: http://www.fao.org/docrep/ w6562s/w6562s $00 . h t m$

3. Chauca L. 2007. Realidad y perspectiva de la crianza de cuyes en los países andinos. En: XX Reunión ALPA. Cusco: Asociación Latinoamericana de Producción Animal.

4. Clavijo R, Loui C, Andersen G, Riley $L, L u S$. 2006. Identification of genes associated with survival of Salmonella enterica serovar Enteritidis in chicken egg albumen. Appl Environ Microbiol 72: 1055-1064. doi: 10.1128/AEM.72.2.1055-1064.2006

5. Eriksson E, Aspan A. 2007. Comparison of culture, ELISA and PCR techniques for salmonella detection in faecal samples for cattle, pig and poultry. BMC Vet Res 22: 3-21. doi: 10.1186/ 1746-6148-3-21

6. [FDA] Food and Drug Administration. 2007. Bacterioogical analytical manual: Salmonella. $8^{\text {th }}$ ed. USA: FDA. $14 \mathrm{p}$.

7. Feder I, Nietfeld J, Galland J, Yeary T, Sargeant J, Oberst R, Tamplin M, Luchansky J. 2001. Comparison of cultivation and PCR-hybridization for detection of Salmonella in porcine fecal and water samples. J Clin Microbiol 39: 2477-2484. doi: 10.1128/ JCM.39.7.2477-2484.2001

8. Ferraz-Castagna S, Muller M, Macagnan M, Rodenbusch $C$, Canal C, Cardoso M. 2005. Detection of Salmonella sp from porcine origin: a comparison between a PCR method and standard microbiological techniques. Braz J Microbiol 36: 373-377. doi: 10.1590/S1517-83822005000400013

9. Fox J, Cohen B, Loew F. 1984. Laboratory animal medicine. Orlando, USA: Academic Press. 766 p.

10. García C. 2011. Salmonelosis porcina en España: prevalencia, factores de riesgo y resistencia antimicrobiana. Tesis doctoral. León, España: Universidad de León. 197 p.

11. González A, Falcón N. 1999. Análisis de datos en Medicina Veterinaria. Publ Tec FMV-UNMSM N. ${ }^{\circ}$ 49: 57-58.

12. González J, Pereira $N$, Soto $Z$, Hernández E, Villarreal J. 2014. Aislamiento microbiológico de Salmonellas pp y herramientas moleculares para su detección. Salud Uninorte 30(1): 73-94.

13. [INEI] Instituto Nacional de Estadística e Informática. 2012. IV Censo Nacional Agropecuario 2012. [Internet]. Disponible en: http://censos.inei.gob.pe/ cenagro/tabulados/

14. [ISO] International Organization for Standardization. 2002. ISO 6579:2002

(E) Microbiology of food and animal feeding stuffs - Horizontal method for the detection of Salmonella spp. $4^{\circ}$ ed. Switzerland: ISO. $27 \mathrm{p}$.

15. Jamshidi A, Kalidari G, Hedayati M. 2010. Isolation and identification of Salmonella Enteritidis and Salmonella Typhimurium from the eggs of retail stores in Mashhad, Iran using conventional culture methods and multiplex PCR assay. J Food Safety 30: 558-568. doi: $10.1111 / \mathrm{j} .1745-$ 4565.2010.00225.x

16. Kanki M, Sakata J, Taguchi M, Kumeda Y, Ishibashi M, Kawai T, Kawatsu K, et al. 2009. Effect of sample preparation and bacterial concentration on Salmonella enterica detection in poultry meat using culture methods and PCR assaying of preenrichment broths. Food Microbiol 26: 1-3. doi: 10.1016/j.fm.2008.07.010

17. Kumar R, Surendran PK, Thampuran N. 2007. Evaluation of culture, ELISA and PCR assays for the detection of Salmonella in seafood. Lett App1 Microbiol 46: 221-226. doi: 10.1111/ j.1472-765X.2007.02286.x

18. Landis J, Koch G. 1977. The measurement of observer agreement for categorical data. Biometrics. 33: 159174. doi: $10.2307 / 2529310$ 
19. Layme A. 2009. Frecuencia de lesiones anatomopatológicas en cobayos con diagnóstico bacteriológico de Salmonella sp remitidos al laboratorio de histología, embriología y patología veterinaria de la FMV-UNMSM durante el periodo 20012007. Tesis de Médico Veterinario. Lima: Univ Nacional Mayor de San Marcos. $66 \mathrm{p}$.

20. Mainar-Jaime RC, Andres J, Vico JP, San Roman B, Garrido V, Grilló M. 2013. Sensitivity of the ISO 6579:2002/ Amd 1:2007 standard method for detection of Salmonella spp on mesenteric lymph nodes from slaughter pigs. J Clin Microbiol 51: 89-94. doi: 10.1128/JCM.02099-12

21. Malorny B, Hoorfar J, Bunge C, Helmuth R. 2003. Multicenter validation of the analytical accuracy of Salmonella PCR: towards an international standard. Appl Environ Microbiol 69: 290-296. doi: 10.1128/ AEM.69.1.290-296.2003

22. Marathe S, Chowdhury R, Bhattacharya $R, \quad$ Nagarajan, Chakravortty. 2012. Direct detection of Salmonella without pre-enrichment in milk, ice-cream and fruit juice by PCR against hilA gene. Food Control 23: 559563. doi: 10.1016/j.foodcont.2011.08.005

23. Marcelo G. 2015. Identificación de Salmonella Enteritidis y Typhimurium aislada de cuyes mediante la técnica de reacción en cadena de la polimerasa múltiple. Tesis de Médico Veterinario. Lima: Univ Nacional Mayor de San Marcos. 51 p.

24. Matias BG, Pinto P, Cossi M, Silva A, Vanetti M, Nero L. 2010. Evaluation of polymerase chain reation protocol for the detection of Salmonella species directly from superficial samples of chicken carcasses and preenrichment broth. Poult Sci 89: 1524-1529. doi: 10.3382/ ps.2009-00368

25. Matsuura A, Morales S, Calle S, Ara M. 2010. Susceptibilidad a antibacterianos in vitro de Salmonella enterica aislada de cobayos de crianza familiar- comercial en la provincia de Carhuaz, Ancash. Rev Inv Vet Perú 21: 93-99. doi: 10.15381/rivep.v21i1.355

26. Myint M, Johnson Y, Tablante N, Heckert R. 2006. The effect of the preenrichment protocol on the sensitivity and specificity of PCR for detection of naturally contaminated Salmonella in raw poultry compared to conventional culture. Food Microbiol 23: 599-604. doi: 10.1016/j.fm.2005.09.002

27. Ocepek M, Pate M, Micunovic J, Bole-Hribovsek V. 2006. Comparison and optimization of two PCR tests for identification of Salmonella in poultry feed-stuffs, liver and faeces. Slov Vet Res 43: 61-66.

28. [OIE] Organización Mundial de Sanidad Animal. 2008. Validación y control de calidad de los métodos de la reacción en cadena de la polimerasa utilizados para el diagnóstico de las enfermedades infecciosas. En: Manual de las pruebas de diagnóstico y de las vacunas para los animales terrestres. $6^{\circ}$ ed. Francia: OIE.

29. [OIE] Organización Mundial de Sanidad Animal. 2010. Salmonellosis. In: Manual of diagnostic tests and vaccines for terrestrial animals. $6^{\text {th }}$ ed. France: OIE.

30. Pérez C, Sanchez M, Henao S, Cardona-Castro N. 2008. Estandarización y evaluación de dos pruebas de reacción en cadena de la polimerasa para el diagnóstico de Salmonella enterica subespecie enterica en huevos. Arch Med Vet 40: 235-242. doi: 10.4067/S0301732X2008000300003

31. Rahn K, De Grandis S, Clarke R, McEwen A, Galan J, Ginocchio C, Curtiss R, Gyles C. 1992. Amplification of an InvA gene sequence of Salmonella Typhimurium by polymerase chain reaction as a specific method of detection of Salmonella. Moll Cell Probes 6: 271279. doi: 10.1016/0890-8508(92)90002-F

32. Ramírez A. 1972. Estudio bacteriológico y epidemiológico de un brote infeccioso en cobayos. Tesis de Médico Ve- 
terinario. Lima: Univ Nacional Mayor de San Marcos. 48 p.

33. [SENASA] Servicio Nacional de Sanidad Agraria. 2014. Procedimiento: recolección y envío de especímenes/ muestras, y exámenes solicitados al laboratorio. [Internet]. Disponible en: http:/ /www.senasa.gob.pe/senasa/wpcontent/uploads/2014/12/PRO-UCDSA06-Recoleccion-y-envio-de-especimenes.pdf

34. Soria MC, Soria MA, Bueno D. 2012. Comparison of 2 culture methods and PCR assays for Salmonella detection in poultry feces. Poult Sci 91: 616-626. doi: 10.3382/ps.2011-01831
35. Soumet C, Ermel G, Rose V, Rose N, Drouin P, Salvat G, Colin P. 1999. Identification by a multiplex PCR-based assay of Salmonella Typhimurium and Salmonella Enteritidis strains from environmental swabs of poultry houses. Lett Appl Microbiol 29: 1-6. doi: 10.1046/ j.1365-2672.1999.00559.x

36. Zhang G, Brown E, Gonzalez-Escalona N. 2011. Comparison of real-time PCR, reverse transcriptase real-time PCR, loop-mediated isothermal amplification, and the FDA conventional microbiological method for the detection of Salmonella spp in produce. Appl Environ Microbiol 77: 6495-6501. doi: 10.1128/AEM.00520-11 